\title{
Empagliflozin modulates renal sympathetic and heart rate baroreflexes in a rabbit model of diabetes
}

\author{
Cindy Gueguen ${ }^{1} \cdot$ Sandra L. Burke ${ }^{1} \cdot$ Benjamin Barzel $^{1} \cdot$ Nina Eikelis $^{2} \cdot$ Anna M. D. Watson $^{3} \cdot$ Jay C. Jha ${ }^{3}$. \\ Kristy L. Jackson ${ }^{1} \cdot$ Yusuke Sata ${ }^{1,4} \cdot$ Kyungjoon Lim $^{1,5} \cdot$ Gavin W. Lambert $^{2} \cdot$ Karin A. M. Jandeleit-Dahm ${ }^{3,6}$. \\ Mark E. Cooper ${ }^{3,7} \cdot$ Merlin C. Thomas $^{3} \cdot$ Geoffrey A. Head ${ }^{1,8}$ (D)
}

Received: 21 January 2020 / Accepted: 10 March 2020 / Published online: 5 May 2020

(C) Springer-Verlag GmbH Germany, part of Springer Nature 2020

\begin{abstract}
Aims/hypothesis We determined whether empagliflozin altered renal sympathetic nerve activity (RSNA) and baroreflexes in a diabetes model in conscious rabbits.

Methods Diabetes was induced by alloxan, and RSNA, mean arterial pressure (MAP) and heart rate were measured before and after 1 week of treatment with empagliflozin, insulin, the diuretic acetazolamide or the ACE inhibitor perindopril, or no treatment, in conscious rabbits.

Results Four weeks after alloxan administration, blood glucose was threefold and MAP 9\% higher than non-diabetic controls $(p<0.05)$. One week of treatment with empagliflozin produced a stable fall in blood glucose $(-43 \%)$ and increased water intake $(+49 \%)$ but did not change RSNA, MAP or heart rate compared with untreated diabetic rabbits. The maximum RSNA to hypotension was augmented by $75 \%(p<0.01)$ in diabetic rabbits but the heart rate baroreflex was unaltered. Empagliflozin and acetazolamide reduced the augmentation of the RSNA baroreflex $(p<0.05)$ to be similar to the non-diabetic group. Noradrenaline (norepinephrine) spillover was similar in untreated diabetic and non-diabetic rabbits but twofold greater in empagliflozin- and acetazolamide-treated rabbits $(p<0.05)$.

Conclusions/interpretation As empagliflozin can restore diabetes-induced augmented sympathetic reflexes, this may be beneficial in diabetic patients. A similar action of the diuretic acetazolamide suggests that the mechanism may involve increased sodium and water excretion.
\end{abstract}

Keywords Baroreflex · Diabetes $\cdot$ Empagliflozin $\cdot$ Perindopril $\cdot$ Renal sympathetic nerve activity $\cdot$ SGLT2 inhibitor

Cindy Gueguen and Sandra L. Burke are joint first authors. Merlin C. Thomas and Geoffrey A. Head are joint senior authors.

Electronic supplementary material The online version of this article (https://doi.org/10.1007/s00125-020-05145-0) contains peer-reviewed but unedited supplementary material, which is available to authorised users.

Geoffrey A. Head

geoff.head@baker.edu.au

1 Neuropharmacology Laboratory, Baker Heart and Diabetes Institute, P.O. Box 6492, Melbourne, VIC 3004, Australia

2 Iverson Health Innovation Research Institute and School of Health Science, Swinburne University of Technology, Melbourne, VIC, Australia

3 Department of Diabetes, Central Clinical School, Monash University, Melbourne, VIC, Australia
4 Central Clinical School, Faculty of Medicine, Nursing and Health Sciences, Monash University, Melbourne, VIC, Australia

5 Department of Physiology, Anatomy \& Microbiology, La Trobe University, Melbourne, VIC, Australia

6 Diabetic Nephropathy Research Group, Institute for Clinical Diabetology, German Diabetes Center (DDZ), Leibnitz Center for Diabetes Research, Heinrich Heine University, Düsseldorf, Germany

7 Baker Heart and Diabetes Institute, Melbourne, VIC, Australia

8 Department of Pharmacology, Monash University, Melbourne, VIC, Australia 


\section{Research in context}

\section{What is already known about this subject?}

- Inhibitors of sodium-glucose cotransporter 2 (SGLT2) reduce cardiovascular risk in people with type 2 diabetes

\section{What is the key question?}

- Are the beneficial effects of the SGLT2 inhibitor empagliflozin associated with a modification in sympathetic nervous system activity or baroreflexes?

\section{What are the new findings?}

- Empagliflozin reduced the exaggerated sympathetic response to lowering blood pressure observed in diabetic rabbits to the level observed in non-diabetic rabbits, effectively normalising the baroreflex. This was not mediated by glucose lowering, as comparable glucose lowering with insulin failed to modulate baroreflexes

- The proximal tubule natriuretic acetazolamide also suppressed the renal sympathetic baroreflex, suggesting that proximal sodium reabsorption regulates this pathway

- Plasma noradrenaline and noradrenaline spillover were elevated after empagliflozin and acetazolamide, but this is likely to reflect sympathetic activity in vascular beds other than renal and cardiac, as renal sympathetic nerve activity and heart rate were not different

\section{How might this impact on clinical practice in the foreseeable future?}

- SGLT2 inhibitors such as empagliflozin may be used to moderate the exaggerated responsiveness of the sympathetic nervous system in diabetic patients

$\begin{array}{ll}\text { Abbreviations } \\ \text { MAP } & \text { Mean arterial pressure } \\ \text { Nu } & \text { Normalised units } \\ \text { RSNA } & \text { Renal sympathetic nerve activity } \\ \text { SGLT2 } & \text { Sodium-glucose cotransporter 2 } \\ \text { SNA } & \text { Sympathetic nerve activity } \\ \text { SNS } & \text { Sympathetic nervous system }\end{array}$

\section{Introduction}

Individuals with diabetes have an increased risk of developing cardiovascular disease which increases their morbidity and mortality. A new generation of glucose-lowering drugs have been developed that increase sodium and glucose excretion in urine by inhibiting the sodium-glucose cotransporter 2 (SGLT2). In patients with type 2 diabetes, treatment with SGLT2 inhibitors such as empagliflozin has been reported to reduce cardiovascular mortality [1,2]. SGLT2 inhibitors block glucose and sodium reabsorption in the close proximal tubules of the renal nephron where SGLT2 is primarily expressed. Excessive resorption of sodium/glucose in the proximal tubules leads to activation of renal afferent nerves and results in central sympathetic overactivity in individuals with poorly controlled type 2 diabetes in whom sympathetic nervous system (SNS) activation is also associated with cardiovascular outcomes [3]. We hypothesise that through their actions in the proximal tubule, SGLT2 inhibitors also modulate the renal
SNS. Selective SGLT2 inhibition has been shown to reduce the power of low-frequency fluctuations in systolic BP in a rat model of the metabolic syndrome displaying hypertension, diabetes and hyperlipidaemia, which has been interpreted as a reduction in sympathetic vasomotor tone [4].

Currently, the effect of an SGLT2 inhibitor on direct recording of renal sympathetic nerve activity (RSNA) is unknown and thus we assessed the changes in RSNA following alloxan-induced diabetes in conscious rabbits, a model in which RSNA can be compared between groups [5]. We explored the effects of acute and 1 week treatment with SGLT2 inhibitor empagliflozin on blood biochemical, cardiovascular and RSNA baroreflex variables and compared these responses with the effects of insulin, the natriuretic acetazolamide and the ACE inhibitor perindopril.

\section{Methods}

\section{Animals}

Experiments were conducted in 48 male New Zealand white rabbits weighing 2.6-3.3 kg. Experiments were approved by the Alfred Medical Research Education Precinct Animal Ethics Committee and conducted according to the Australian code for the care and use of animals for scientific purposes (2013). Rabbits were housed individually with free access to 
water and food under controlled humidity $(40-70 \%)$ and temperature $\left(22 \pm 2^{\circ} \mathrm{C}\right)$ and a $12 / 12$ light cycle.

\section{Induction of diabetes}

Alloxan [6] (60 mg/kg i.v.; Sigma-Aldrich, Saint-Louis, MO, USA) was injected under brief propofol sedation $(6 \mathrm{mg} / \mathrm{kg}$ i.v.; Fresofol 1\%, Fresenius Kabi, Pymble, NSW, Australia). Blood glucose was monitored 4 hourly during the first $48 \mathrm{~h}$ and animals were supplemented with $25 \%$ glucose when necessary. From day 2, rabbits received long-acting glargine insulin s.c. daily (Lantus, Sanofi, Macquarie Park, NSW, Australia) to maintain their blood glucose in the target range of 15-25 mmol/1 [7]. One rabbit became irreversibly hypoglycaemic after alloxan and one other became unwell during the study, and these were euthanised.

\section{Renal nerve electrode surgery}

After a mean of 22 days, rabbits were implanted with a renal nerve recording electrode [8]. They were anaesthetised with propofol (10 mg/kg i.v.) and maintained under isoflurane (3\% in $1 \mathrm{l} / \mathrm{min}$ oxygen; Forthane, AbbVie, Mascot, NSW, Australia). Carprofen (3 mg/kg; Rimadyl, Zoetis, Rhodes, NSW, Australia) was given before (i.v.) and $24 \mathrm{~h}$ after surgery (s.c.) for analgesia.

\section{Study design}

The effects of treatment were examined over a period of $3 \mathrm{~h}$ after a single acute dose and at the end of repeated daily doses for 1 week. Rabbits were randomly assigned to diabetic empagliflozin (Diab+Empa, $1 \mathrm{mg} / \mathrm{kg}$ acute, $11.3 \mathrm{mg} \mathrm{kg}^{-1}$ day $^{-1}$ chronic, $n=7$ ), insulin (Diab+Insu, 4-5 U acute, $11.5 \mathrm{U} / \mathrm{d}$ chronic, $n=8$ ), acetazolamide (Diab+Acet, $4 \mathrm{mg} / \mathrm{kg}$ acute, $36 \mathrm{mg} \mathrm{kg}^{-1}$ day $^{-1}$ chronic, $n=8$ ), perindopril (Diab+Peri, $0.4 \mathrm{mg} / \mathrm{kg}$ acute, $0.42 \mathrm{mg} \mathrm{kg}^{-1}$ day ${ }^{-1}$ chronic, $n=7$ ), untreated (Diab, $n=8$ ) and non-diabetic (Non-diab, $n=8$ ) groups. See the electronic supplementary material (ESM) Methods and ESM Table 1.

\section{Daily body weight, blood glucose, and water and food intake measurements}

Body weight and blood glucose measurements at day 0 and water and food intake, measured over 3 days before alloxan administration, were used as baseline. All variables were then measured daily before water and food replenishment. To evaluate the effect over 1 week of each treatment drug, the 3 days before treatment/non-treatment were used as that control period. Blood glucose was measured by glucometer (Accu-Chek Performa, Roche, Bella Vista, NSW, Australia) from a drop of blood taken from a superficial ear vein.

\section{Blood biochemistry measurements}

Blood glucose, sodium, chloride, potassium, ionised calcium (iCa), total carbon dioxide $\left(\mathrm{TCO}_{2}\right)$, creatinine, blood urea nitrogen/urea (urea), haemoglobin, haematocrit and anion gap (AnGap) were measured from a blood sample $(0.2 \mathrm{ml})$ using the i-stat system $\left(\mathrm{Chem} 8^{+}\right.$cartridge, Abbott, Melbourne, VIC, Australia) before alloxan administration, before the first treatment drug and at the end of the 1 week treatment period.

\section{Conscious experimental measurements}

One week after electrode surgery, rabbits were placed in single holding boxes in the laboratory and the central ear artery was catheterised to measure mean arterial pressure (MAP) and heart rate [5]. RSNA was rectified and integrated [9]. See the ESM Methods for details. RSNA was normalised by the nasopharyngeal response expressed as 100 normalised units (nu). After 30 min recovery from handling, control measurements were recorded for $60 \mathrm{~min}$ and blood glucose was measured. Rabbits were then given a single dose of the treatment drug and data were recorded over the following $3 \mathrm{~h}$, with blood glucose measured each hour.

For the 1 week treatment, rabbits received the same drug but at a chronic dose (ESM Table 1). After 1 week of treatment, cardiovascular measurements were recorded for $3 \mathrm{~h}$, with hourly blood glucose. RSNA and heart rate baroreflexes were derived from slow ramp rises and falls in MAP by i.v. infusions of $25 \mathrm{mg} / \mathrm{kg}$ phenylephrine (Sigma-Aldrich) and $30 \mathrm{mg} / \mathrm{kg}$ nitroprusside (Fluka AG, Buchs, Switzerland), respectively. Noradrenaline (norepinephrine) spillover was determined using $\left[{ }^{3} \mathrm{H}\right]$-noradrenaline $\left(4070 \mathrm{~Bq} \mathrm{~kg}^{-1} \mathrm{~min}^{-1}\right.$ i.v.; Perkin Elmer Life Sciences, Boston, MA, USA; see ESM Methods). Rabbits were killed with sodium pentobarbitone $(160 \mathrm{mg} / \mathrm{kg}$ i.v.; Lethobarb, Virbac, Milperra, NSW, Australia) and organs removed and weighed.

\section{Baroreflex curves and spectral analysis methods}

MAP, heart rate and RSNA were averaged over $2 \mathrm{~s}$ intervals and fitted to a five-variable sigmoid logistic function to produce MAP-RSNA and MAP-heart rate baroreflex curves as previously described [10]. Variables included the lower plateau (minimum RSNA or heart rate), the range between the lower and upper plateau and the median blood pressure at half the reflex range (BP50). The beat-to-beat instantaneous MAP over the $3 \mathrm{~h}$ of chronic treatment was analysed for spectral analysis [11]. See the ESM Methods for further details.

\section{Confirmation of ACE inhibition}

Rabbits in the ACE inhibitor perindopril treatment group were given a bolus injection of angiotensin I (50-200 pmol, 
Auspep, Tullamarine, VIC, Australia) before and after treatment with perindopril, both acute and chronic, and the changes in MAP were compared to determine whether the doses of perindopril were adequate to block the renin-angiotensin system.

\section{Statistical analysis}

Data were expressed as mean \pm SEM or mean difference \pm standard error of the difference (SED) and analysed by a mixed model multivariate ANOVA followed by Student's $t$ tests to determine the effect of diabetes $\left(p_{\text {diab }}\right)$, differences at baseline $\left(p_{\text {base }}\right)$, and treatment effects compared with control $\left(p_{\text {treat }}\right)$ and between-group treatment effects $\left(p_{\text {delta }}\right)$ with a pooled variance [12]. Family-wise error was controlled by the Bonferroni procedure and multisample asphericity by the Greenhouse-Geisser correction [13]. A probability of $p<0.05$ was considered significant.

\section{Results}

\section{Effects of diabetes on metabolic and cardiovascular variables}

Alloxan induced a stable level of hyperglycaemia with blood glucose $20.5 \pm 0.4 \mathrm{mmol} / \mathrm{l}$ compared with $6.5 \pm 0.4 \mathrm{mmol} / \mathrm{l}$ in non-alloxan rabbits $\left(p_{\text {diab }}<0.001\right)$. Diabetic rabbits gained weight at a slower rate than controls $(+3 \%$ vs $+7 \%$, respectively, $\left.p_{\text {diab }}<0.001\right)$ even though water intake was greatly elevated $\left(+55 \%\right.$ vs $\left.-17 \%, p_{\text {diab }}<0.001\right)$ and food consumption was greater $\left(p_{\text {diab }}=0.045\right.$; ESM Fig. 1$)$. The MAP of diabetic rabbits was $9 \%$ greater than non-diabetic $\left(p_{\text {diab }}=\right.$ 0.016; Fig. 1). There was no effect of diabetes on heart rate, total RSNA, burst amplitude, burst incidence or burst frequency (Fig. 1). Plasma sodium and chloride were reduced by 5 $6 \%$ compared with non-diabetic animals $\left(p_{\text {diab }}<0.001 ;\right.$ ESM Table 2) but potassium and other blood biochemistry measures were similar (ESM Table 2).

\section{Acute effects of treatments on blood glucose, cardiovascular variables and RSNA}

After establishment of diabetes, rabbits underwent acute treatment with empagliflozin, insulin, acetazolamide, perindopril or vehicle and the effects were followed for $3 \mathrm{~h}$. In the vehicletreated group, blood glucose and MAP remained stable over $3 \mathrm{~h}$ after vehicle injection and not different from the control period ( $p_{\text {treat }}>0.05$; Fig. 2 ), but heart rate and total RSNA increased $\left(+13 \%\right.$ and $+26 \%$, respectively, $p_{\text {treat }}<0.05$; Fig. 2, ESM Fig. 2, ESM Table 3).

Empagliflozin induced a fall in blood glucose of $-5.5 \pm$ $1.2 \mathrm{mmol} / 1$ over $3 \mathrm{~h}\left(-31 \%, p_{\text {treat }}<0.001, n=7\right.$; Fig. 2). There was little change in MAP but we observed linear
Fig. 1 Effects of diabetes on cardiovascular and RSNA variables. (a) MAP, (b) heart rate and (c-h) RSNA in non-diabetic (Non-diab, $n=8$ ) and diabetic (Diab, $n=39$ ) rabbits 4 weeks after alloxan treatment. RSNA shown as (c) RSNA burst incidence, $(\mathbf{d}, \mathbf{g})$ total RSNA and (e, h) burst amplitude (amp), expressed in normalised units (nu) and microvolts $(\mu \mathrm{V})$, and (f) burst frequency (freq). (i) Nasopharyngeal response to smoke is also shown (numbers are the same as for $\mathbf{a}-\mathbf{h}$ ). Data are mean $\pm \mathrm{SEM}$, indicating betweenanimal variance from the $1 \mathrm{~h}$ control period before acute treatment. $* p<0.05$ for diabetic vs non-diabetic a
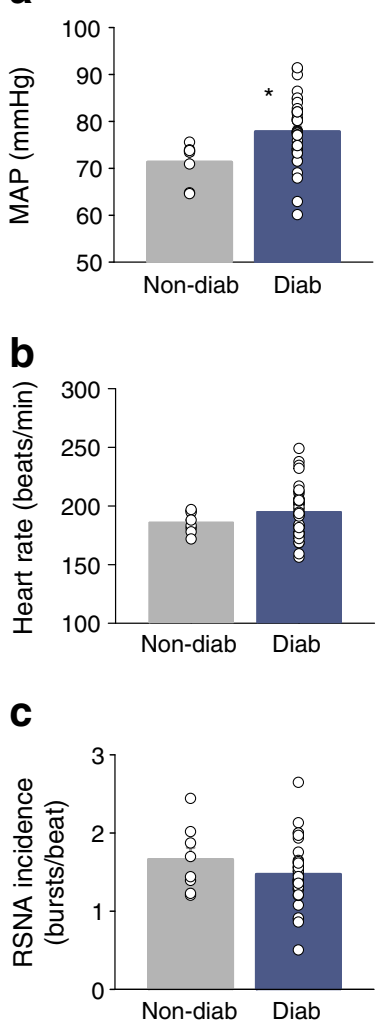

d

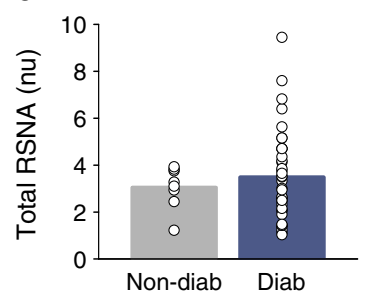

e

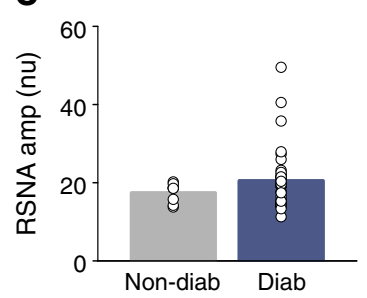

f

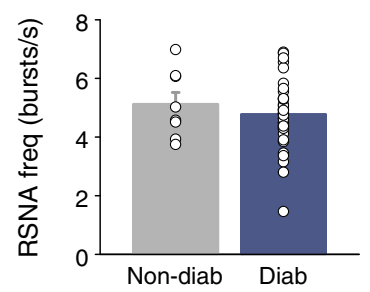

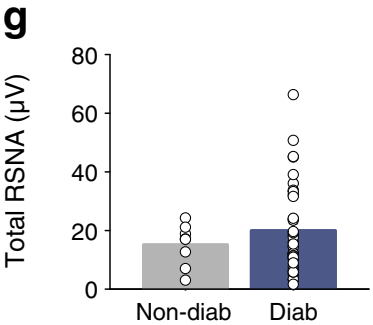

h

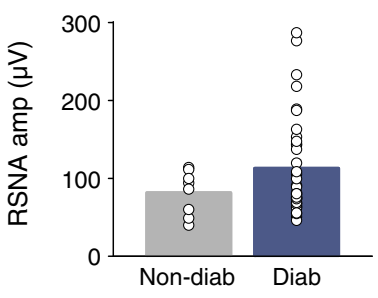

i

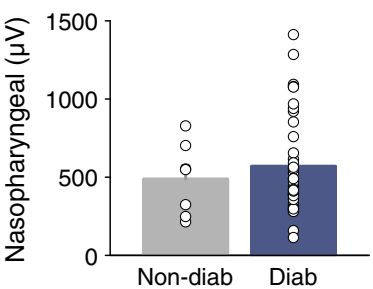


Fig. 2 Time course of effects of treatment over $3 \mathrm{~h}$ on (a) blood glucose level, (b) MAP, (c) heart rate and (d) total RSNA in diabetic rabbits. (e-h) Mean change from control $(\mathrm{C} 1-\mathrm{C} 2)$ over $3 \mathrm{~h}$ and $(\mathbf{i}-\mathbf{l})$ mean change from control after 1 week of chronic treatment. Groups are vehicle (V, dark blue; $n=8$ ), untreated (U, dark blue; $n=8$ ), empagliflozin (E, red; $n=8$ ), insulin (I, purple; $n=8$ ), acetazolamide (A, pink; $n=8$ ) and perindopril (P, light blue; $n=$ 7) in diabetic rabbits. Error bars are SEM, indicating betweenanimal variance. $* * * p_{\text {delta }}<0.001$ and ${ }^{*} p_{\text {delta }}<0.01$ compared with control group. $\mathrm{C} 1$, average first 30 min of control period; $\mathrm{C} 2$, average second $30 \mathrm{~min}$ of control period
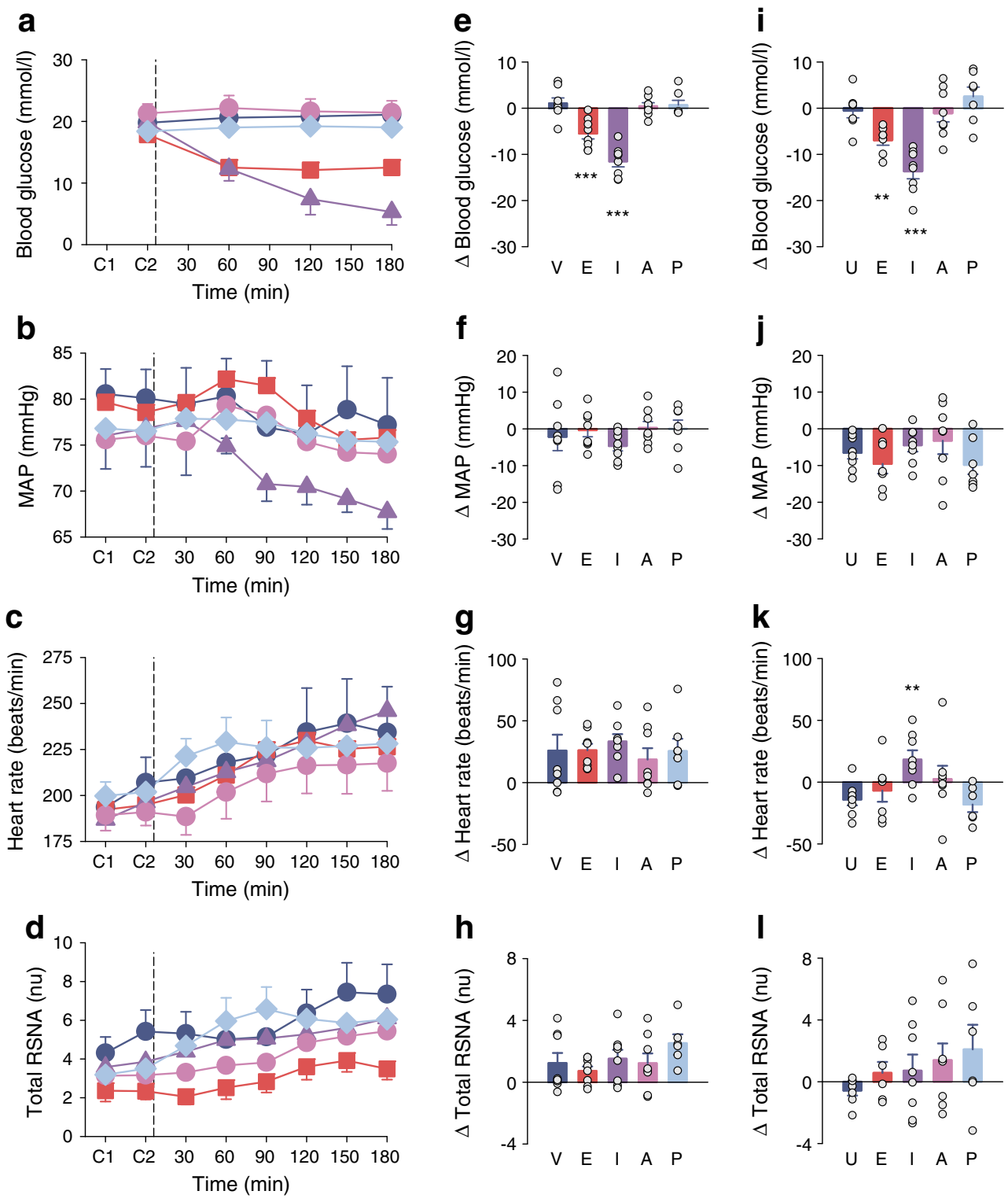

h

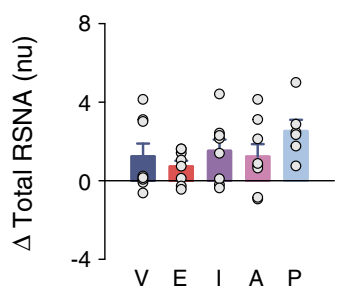

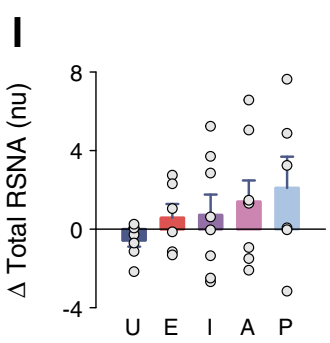

increases in heart rate and RSNA (total RSNA, burst incidence and frequency, all $p_{\text {linearity }}<0.001$ ), similar to the effect of vehicle (ESM Table 3). Treatment with insulin lowered blood glucose over $3 \mathrm{~h}\left(-12.4 \pm 2.2 \mathrm{mmol} / 1, p_{\text {treat }}<0.001\right.$, $n=8)$. There was a linear fall in MAP of $6 \%$ accompanied by increases in heart rate $(17 \%)$ and total RSNA $\left(41 \%, p_{\text {treat }}\right.$ $<0.001$ ), which was similar to vehicle treatment (Fig. 2). However, MAP in the insulin-treated rabbits was lower after $3 \mathrm{~h}$ than the MAP in non-diabetic controls $(68 \pm 2 \mathrm{mmHg}$ vs $73 \pm 2 \mathrm{mmHg}, p=0.022)$ despite similar blood glucose. We observed a linear relationship between change in blood glucose and fall in MAP in the insulin group $(r=0.57$, $p<0.001$ ), but not in the empagliflozin group (ESM Fig. 3). Acetazolamide and perindopril administration had no observable effects on blood glucose or MAP ( $\left.p_{\text {treat }}>0.05\right)$ but heart rate increased by $10 \%$ and $13 \%$, respectively (both $p_{\text {treat }}$
$<0.001$ ), and RSNA in response to acetazolamide increased by $39 \%$ ( $p_{\text {treat }}<0.01$ ), all similar to the responses to vehicle (Fig. 2, ESM Table 3). In the non-diabetic group, MAP, heart rate and total RSNA remained stable over the $3 \mathrm{~h}$ recording period (all $p_{\text {treat }}>0.05$; ESM Fig. 2).

\section{Differences between treatment groups in control period for acute treatment}

In diabetic rabbits, there were no differences in blood glucose, MAP and heart rate between treatment groups and the vehicletreated group during the control period (ESM Table 4). Whilst control total RSNA was $52 \%$ lower in the empagliflozin group compared with the vehicle-treated group (ESM Table 4), it remained 50\% lower throughout the treatment period (mean over 3 h: $3.1 \pm 0.5$ vs $6.1 \pm 1.2 \mathrm{nu}$, respectively, $p=0.005$ ). 
There were no differences in control total RSNA between the untreated diabetic group and the other treatment groups (ESM Table 4).

\section{One week effects of empagliflozin, insulin, acetazolamide and perindopril}

Cardiovascular variables and RSNA Diabetic rabbits were treated for 1 week with empagliflozin, insulin, acetazolamide or perindopril, or were untreated for that period. Between the acute and chronic recording days for the non-diabetic group, there was an increase in MAP of $6 \%\left(p_{\text {treat }}=0.014\right)$, but in this group neither heart rate nor total RSNA changed between the two experiments (ESM Fig. 4). However, all diabetic animals decreased MAP and heart rate over the week between the two recording days and there was no difference among groups for MAP or RSNA. In untreated diabetic rabbits, mean MAP and heart rate decreased by $8 \%$ and $7 \%$, respectively (both $p_{\text {treat }}<0.001$; Fig. 2 ). Total RSNA was also $13 \%$ lower $\left(p_{\text {treat }}=0.022\right)$ due to a reduction in burst amplitude $(-32 \%$, $p_{\text {treat }}<0.001$; ESM Table 3$)$. The fall in MAP and the stable heart rate and total RSNA after 1 week empagliflozin treatment were not different from those in untreated diabetic rabbits (Fig. 2), although there was an increase in RSNA amplitude (ESM Table 3). Similarly, we observed no difference between insulin-treated and untreated groups in MAP and total RSNA changes but there was a $10 \%$ increase in heart rate compared with the untreated group in which heart rate decreased $\left(p_{\text {delta }}=0.003\right.$; Fig. 2$)$. Acetazolamide did not alter the pattern observed in untreated rabbits, but after perindopril treatment MAP was $9 \%$ lower than in the untreated group $(p=0.045$; Fig. 2)

Blood glucose, body weight, and water and food intake In the untreated diabetic group, blood glucose, body weight, and water and food intake remained stable and similar to the control levels over the 1 week period (ESM Fig. 5). Empagliflozin treatment over 1 week produced a stable decrease in blood glucose $\left(43 \%, p_{\text {treat }}<0.001\right)$ without requiring insulin to prevent hyperglycaemia. Water consumption increased by $49 \%\left(p_{\text {treat }}<0.001\right)$, markedly greater than changes in water intake in untreated diabetic rabbits $\left(p_{\text {delta }}<0.001\right.$; ESM Fig. 5). There was a small but significant $2 \%$ loss of body weight during empagliflozin treatment $\left(p_{\text {treat }}=0.001\right)$ but the drug did not alter food intake (ESM Fig. 5). In the insulin-treated group, blood glucose gradually declined so that by day 7 it was $32 \%$ lower than control $\left(p_{\text {linearity }}<0.001\right)$ but similar to the empagliflozin-treated group (ESM Fig. 6). Weight gain in insulin-treated rabbits $\left(+2 \%, p_{\text {treat }}<0.001\right)$ was greater than in untreated rabbits $\left(p_{\text {delta }}<0.001\right)$ despite food intake not changing, but water intake was reduced by $26 \%\left(p_{\text {treat }}<0.001, p_{\text {delta }}=0.024\right.$; ESM Fig. 5). In the acetazolamide group, the ongoing treatment with insulin maintained blood glucose at a stable level over 1 week (ESM Fig. 6), but water and food intake both fell markedly over the course of 7 days $(-44 \%$ and $\left.-35 \%, p_{\text {treat }}<0.001\right)$. This was associated with a $3 \%$ loss of body weight ( $p_{\text {treat }}<0.001$; ESM Fig. 5). One week treatment with perindopril had no observable effects on blood glucose, water or food consumption and body weight (all $p_{\text {treat }}>0.05$; ESM Figs. 5, 6). In non-diabetic rabbits, there was a small but significant $1 \%$ weight gain over 1 week $\left(p_{\text {treat }}<0.001\right)$.

Blood biochemistry In the untreated diabetic group, we observed no change in blood biochemistry after the 1 week period. Empagliflozin, insulin or perindopril treatment was not associated with any differences in biochemical measurements compared with untreated diabetic rabbits (ESM Table 5). Acetazolamide lowered potassium levels by $-17 \%$, a greater effect than in untreated diabetic rabbits $\left(p_{\text {delta }}=\right.$ 0.012 ), and increased blood urea concentrations (+29\%, $p$ delta $=0.042$ vs untreated; ESM Table 5), but did not affect any other blood variables. Blood biochemical variables remained stable over 1 week in the non-diabetic group (ESM Table 6).

Organ weights Kidney weight, normalised to body weight, was $20 \%$ greater in the untreated diabetic group compared with the non-diabetic group. We observed no additional effects of any of the 1 week treatment drugs (ESM Table 7). There were no detectable effects on either whole heart or left ventricular weight of diabetes or any of the treatment drugs (ESM Table 7).

\section{Effects of diabetes and 1 week treatments on RSNA and heart rate baroreflexes}

Effects of diabetes The main effect of diabetes on RSNA baroreflex curves was to markedly increase the upper plateau by $75 \%$, range $(+69 \%)$ and gain $\left(+87 \%, p_{\text {diab }}<0.01\right.$; ESM Fig. 7). A similar effect was seen in the upper plateau and range of the RSNA amplitude curves but the upper plateau and range of the RSNA frequency curves were inhibited. Heart rate baroreflex curves were similar in the diabetic and non-diabetic groups (ESM Fig. 7).

Effects of 1 week treatment Empagliflozin reduced both the RSNA upper plateau and range by $34 \%\left(p_{\text {diab }}=0.016\right)$ such that they were similar to non-diabetic rabbits (Fig. 3). A similar effect was observed after acetazolamide treatment, with $\sim 30 \%$ reductions $\left(p_{\text {diab }}<0.05\right.$; Fig. 3 ). Empagliflozin also reduced the heart rate baroreflex upper plateau $\left(p_{\text {diab }}=\right.$ 0.046; Fig. 3). There was little effect of these drugs on RSNA burst frequency curves (ESM Table 8). There were no effects of insulin or perindopril on either the RSNA or heart rate baroreflex. 

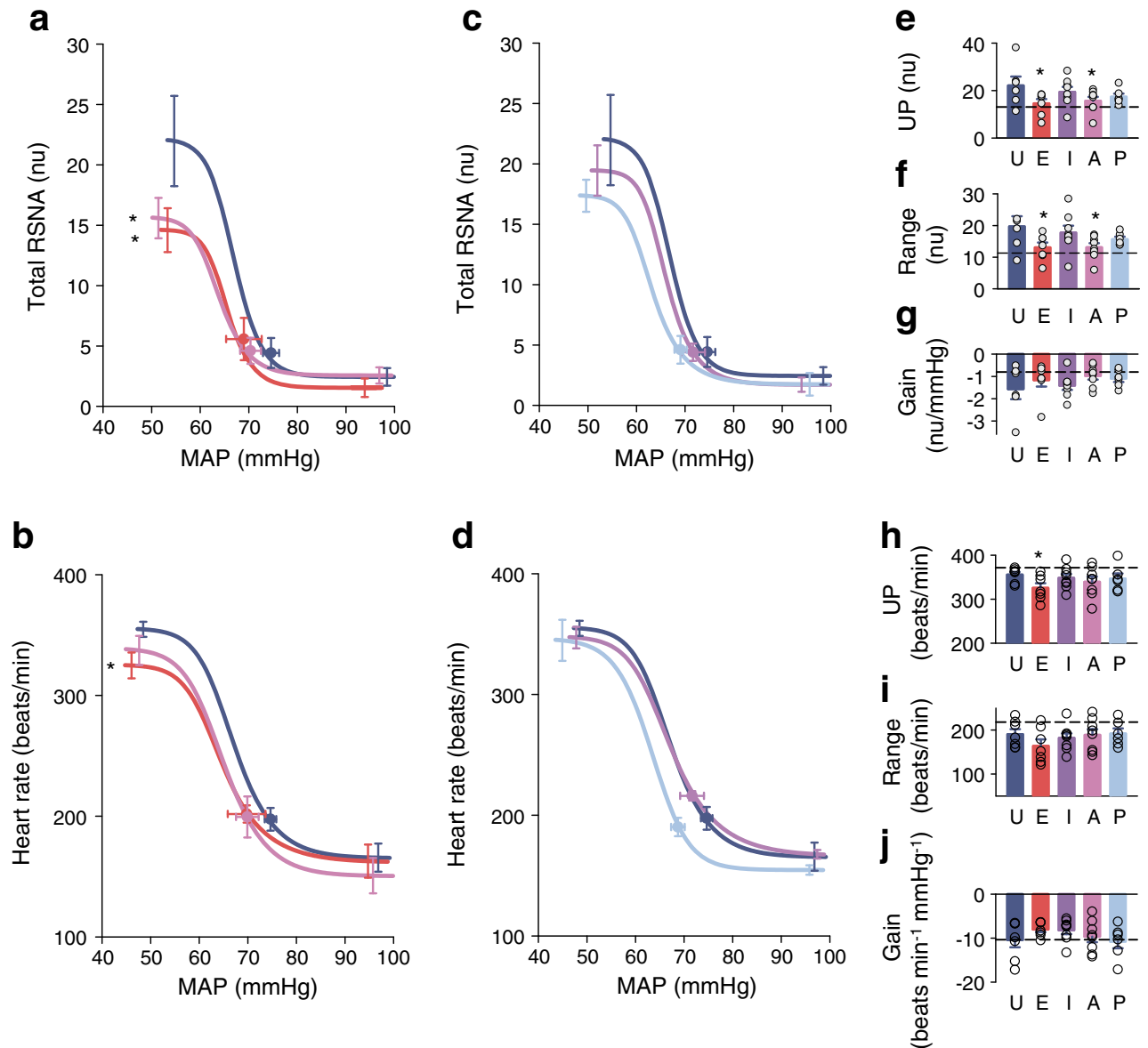

Fig. 3 Effects of 1 week drug treatment on total RSNA and heart rate baroreflex curves in diabetic rabbits. (a) Total RSNA curves from rabbits treated with empagliflozin (red, $n=7$ ) and acetazolamide (pink, $n=8$ ) and untreated (dark blue, $n=7$ ); (b) heart rate baroreflex curves from the same rabbits; (c) total RSNA curves from rabbits treated with insulin (purple, $n=7$ ) and perindopril (light blue, $n=8$ ) and untreated (dark blue, $n=7)$; and (d) heart rate baroreflex curves from the same rabbits. RSNA data were normalised to the nasopharyngeal response in each rabbit.

\section{Effect on short-term cardiovascular variability}

Low, mid, high and total power of MAP and heart rate were similar in non-diabetic and diabetic rabbits (ESM Table 9). In empagliflozin-treated rabbits, MAP power in all bands and low and high heart rate power were similar to diabetic controls. By contrast, mid-frequency heart rate power was elevated compared with controls (ESM Table 9). Insulintreated rabbits exhibited reduced mid- and high-frequency MAP power and reduced high-frequency heart rate power compared with untreated diabetic controls (ESM Table 9). Acetazolamide treatment did not affect any MAP power but low- and mid-frequency heart rate power was elevated compared with controls (ESM Table 9). Perindopril-treated rabbits had lesser low-, mid- and high-frequency and total MAP power and reduced high-frequency heart rate power compared with untreated diabetic rabbits. Baroreflex gain
Symbols on curves represent resting values. Bar graphs show three of the curve variables, upper plateau (UP, $\mathbf{e}, \mathbf{h})$, range (f, i) and gain $(\mathbf{g}, \mathbf{j})$ after empagliflozin (E, red bars), insulin (I, purple bars), acetazolamide (A, pink bars) or perindopril ( $\mathrm{P}$, light blue bars) or untreated (U, dark blue bars). Reference values in non-diabetic rabbits are shown as horizontal dashed lines. Error bars are SEM, indicating between-animal variance. $* p<0.05$ for treated vs untreated rabbits

estimated by cross-spectral analysis was similar in all groups (ESM Table 9). Thus, the pattern of effects observed in the empagliflozin group more closely aligns with the effects of acetazolamide than with insulin or perindopril.

\section{Effects of diabetes and 1 week treatments on noradrenaline kinetics}

Noradrenaline plasma level, spillover to plasma and clearance were similar in diabetic and non-diabetic control rabbits ( $p>0.05$; Fig. 4). However, spillover was elevated twofold after 1 week treatment with empagliflozin $\left(+147 \%, p_{\text {diab }}=0.006\right)$ and acetazolamide $\left(+134 \%, p_{\text {diab }}=\right.$ $0.014)$. Plasma noradrenaline was higher after empagliflozin compared with diabetic and non-diabetic rabbits $\left(p_{\text {non-diab }}<0.01\right.$; Fig. 4$)$. 

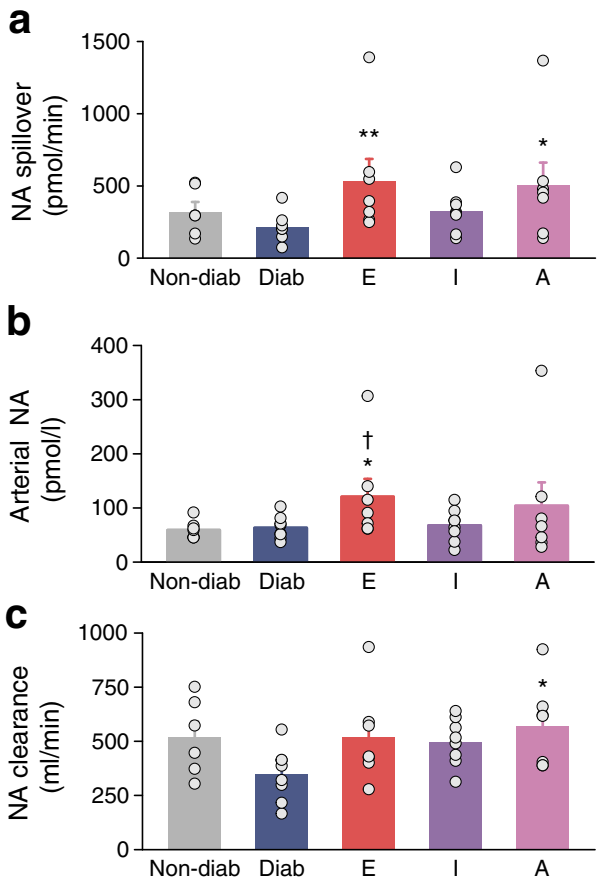

Fig. 4 Noradrenaline kinetics in non-diabetic and diabetic rabbits. (a) Noradrenaline (NA) spillover to plasma, (b) noradrenaline arterial concentration and (c) noradrenaline clearance were measured in nondiabetic (Non-diab, grey bars, $n=8$ ) and untreated diabetic (Diab, blue bars, $n=7$ ) rabbits and in diabetic rabbits treated for 1 week with empagliflozin (E, red bars, $n=7$ ), insulin (I, purple bars, $n=8$ ) or acetazolamide (A, pink bars, $n=7$ ). Data are mean \pm SEM, indicating between-animal variance. $* p<0.05, * * p<0.01$ for treated diabetic vs untreated diabetic rabbits; ${ }^{\dagger} p<0.05$ for treated diabetic vs untreated non-diabetic rabbits

\section{Effects of angiotensin I}

To confirm the inhibition of ACE by perindopril, angiotensin I was administered after cardiovascular control period recording and at the end of the $3 \mathrm{~h}$ recording. Before perindopril treatment, angiotensin I induced a $60 \%$ increase in MAP (ESM Fig. 8). After perindopril treatment, MAP was only increased by $25 \%$ after angiotensin I administration, whether after the acute or the chronic treatment doses of perindopril (ESM Fig. 8), confirming ACE inhibition in both cases.

\section{Discussion}

\section{Principal findings and mechanisms}

The present study examined the SNS effects of acute and 1 week treatment effects of SGLT2 inhibition, in alloxaninduced diabetic rabbits with hyperglycaemia and mild hypertension, compared with non-diabetic controls. One week treatment with empagliflozin produced a stable and marked reduction in blood glucose $(-43 \%)$ and an increase in total noradrenaline spillover, but did not change RSNA, MAP or heart rate. The main effect on RSNA was to reduce the exaggerated response to hypotension (baroreflex upper plateau) to a level similar to that seen in non-diabetic controls. These changes were also observed with acetazolamide but not insulin, which indicates that empagliflozin modulation of the SNS is related to its natriuretic rather than its glucose-lowering action. The main effect of empagliflozin on the cardiac baroreflex was a reduction in the upper plateau, which most likely reflects a reduction in cardiac sympathetic nerve activity (SNA) since vagal activity is inhibited and baro-sensitive cardiac SNA is at its greatest at this point on the curve.

This change to baroreflex responsiveness is similar to that induced during mild-intensity exercise [14] and is thought to buffer the BP against vasodilation associated with exercise. Presumably, in early diabetes these same changes would allow BP and single-nephron GFR to be maintained at a higher level. Enhanced muscle SNA responses to exercise and the cold pressor test have been reported in diabetic individuals, and occurred without changes to the gain of the baroreflex [15]. However, it has also been reported that muscle SNA responses to an oral glucose tolerance test are blunted in type 2 diabetic patients [16]. In our model there was greater RSNA baroreflex gain in diabetic compared with non-diabetic rabbits. Thus, for small alterations in BP near the resting level, the responsiveness of RSNA was enhanced. The augmentation of the total integrated RSNA baroreflex in diabetic rabbits could be attributed to an increase in the upper plateau and range of the RSNA burst amplitude baroreflex. The mechanism underlying this may be additional discharges from previously silent presynaptic neurons in the central nervous system, as modulation of burst amplitude reflects recruitment of neurons [17]. With the restoration of the RSNA baroreflex involving reduced sympathetic responsivity (gain), we might have expected an increase in short-term BP variability, but surprisingly there was no effect of empagliflozin or acetazolamide on BP variability. By contrast, the treatments that did not affect the RSNA baroreflex, insulin and perindopril, did reduce short-term BP variability.

The mechanism whereby empagliflozin was able to normalise the RSNA baroreflex remains to be established. SGLT2 plays a key role in sodium balance, reabsorbing from the lumen of the early proximal tubule one molecule of sodium for every glucose molecule. Following SGLT2 inhibition, increased delivery of sodium and glucose also influences tubular function including the absorption of other solutes and regulation of renal blood flow. We speculate that SGLT2 inhibition also modulates renal afferent activity to inhibit sympathetic neurons to not only prevent tachycardia in response to volume lowering, but also to suppress maximal RSNA in response to BP lowering. This is most likely due to changes in renal sodium balance as we observed a similar effect with acetazolamide, a carbonic anhydrase inhibitor that also acts as a proximal tubular natriuretic, partly by 
modulating the activity of the sodium/proton exchanger isoform 3 [18]. Notably, the effect of empagliflozin was not observed with a single dose, suggesting that it represents a realignment of renal function rather than a direct (instantaneous) effect of the drug on RSNA. Treatment with insulin did not alter the RSNA response, despite a similar lowering of blood glucose as for the SGLT2 treatment. ACE inhibition did not change the RSNA in this model, despite its known actions on renal and systemic haemodynamics and renoprotective actions in vivo.

Noradrenaline spillover to plasma was greater in diabetic empagliflozin-treated rabbits than in untreated rabbits. The higher spillover likely accounted for greater arterial concentrations as noradrenaline clearance did not change. Higher noradrenaline release was also observed in animals treated with acetazolamide, suggesting it may be related to its comparable actions on plasma volume, BP and natriuresis. Taken together with the measurement of RSNA and heart rate baroreflexes, the higher spillover is likely to be in other vascular beds (not renal or cardiac) and less critical for long-term cardiovascular outcomes.

\section{Strengths and weaknesses}

A key strength of the current study is the use of alloxaninduced diabetic rabbits, a model of stable chronic hyperglycaemia, and the ability to have RSNA and cardiovascular variables recorded with these animals over several weeks without anaesthesia. Further, RSNA can be calibrated to allow comparison among different groups. We used comparison treatments that allowed us to compare the independent roles of hypoglycaemia, natriuresis and hypotension. We confirmed that acetazolamide had a diuretic effect because levels of urea increased but serum potassium was reduced, consistent with its known diuretic action [19]. We also confirmed that perindopril blocked angiotensin I conversion.

Limitations were that we used only one dose of each agent, that treatment was for only 1 week and that we did not use relevant combination treatments such as SGLT2 together with ACE inhibition. Chronic treatment with SGLT2 inhibitors such as empagliflozin in humans is most commonly associated with a small reduction in BP [20,21]. The effect size in this study was actually similar but was not statistically significant. In a rat model of diabetes, treatment for 7 weeks with empagliflozin had no effect on BP [22] but did increase urine volume and water intake without affecting sodium balance [22]. Clearly, increased water intake is consistent with glycosuria-mediated diuresis [23], but any reduction in blood volume was not enough to lower BP. Also, the effect of empagliflozin in rats was maximal after 3 days and stable for 7 weeks, suggesting that our study lasting 1 week may well reflect longer-term chronic treatment [22].

\section{Meaning of the study}

The lower levels of sympathetic excitation occurring when BP fluctuates may better protect single-nephron GFR by preventing excessive afferent vasoconstriction when the GFR is already reduced. This could also partly explain the reduced risk of acute kidney injury observed in the EMPAREG OUTCOME trial in patients receiving empagliflozin [24]. Our findings may be particularly important for patients with comorbidities including established cardiovascular disease, chronic heart failure and chronic kidney disease, in whom RSNA is often augmented. The reduction in the upper plateau of the cardiac baroreflex may also indicate a beneficial effect of SGLT2 inhibition on reducing cardiac SNA during hypotension, and may be one explanation for the better long-term cardiovascular outcome [25].

\section{Strengths and weaknesses in relation to other studies}

Many studies have indicated that muscle SNA is increased in diabetes [3, 26, 27], and this has been associated with poor clinical outcomes and increased cardiovascular risk and end organ damage $[26,28]$. We hypothesised that SGLT2 inhibition would modulate resting RSNA but this was not the case. We did not observe any differences in plasma noradrenaline, total noradrenaline spillover or clearance in the diabetic compared with non-diabetic rabbits, suggesting that there was no increase in global SNA associated with diabetes. In humans with type 2 diabetes there were no changes in muscle SNA after several days of empagliflozin treatment, despite an increase in urine volume, weight loss and a reduction in BP [29]. In the present study, once RSNA was increased (by hypotension), the normalising effects of empagliflozin were clearly observed, with lower maximal RSNA and heart rate in diabetic rabbits treated with an SGLT2 inhibitor.

\section{Future research}

Clearly, a longer treatment would be an important next step to determine whether these effects on the SNS are sustained. Furthermore, it would be of interest to use this model to explore the end organ protective effects of combination treatments such as SGLT2 and ACE inhibition.

Acknowledgements We acknowledge the excellent technical assistance of S. Phillips (Iverson Health Innovation Research Institute and School of Health Science, Swinburne University of Technology, Australia), A. Dai (Department of Diabetes, Central Clinical School, Monash University, Australia), M. Patterson and J.-L. Moretti (Neuropharmacology Laboratory, Baker Heart and Diabetes Institute, Australia). Illustrations of the kidney and nephron in the Graphical Abstract were drawn using pictures from Servier Medical Art. Servier Medical Art by Servier is licensed under a Creative Commons Attribution 3.0 Unported License (https://creativecommons.org/licenses/by/3.0/). 
Data availability The data that support the findings of this study are available from the corresponding author upon reasonable request.

Funding This work was supported by Boehringer Ingelheim and in part by the Victorian Government's OIS Program. Investigators were supported by a National Health and Medical Research Council (NHMRC) Project Grant to AMDW (GNT1079645), an NHMRC Early Career Fellowship to JCJ (APP1126169), NHMRC Postdoctoral Fellowships to KL (APP 1053928) and KLJ (APP1091688), a National Heart Foundation Fellowship to YS (APP101325), NHMRC Senior Research Fellowships to KAMJ-D (APP 1059124) and MCT (APP107555), an NHMRC Principal Research Fellowship to GAH (APP1002186) and an NHMRC Senior Principal Research Fellowship to MEC (APP1078808). GWL and NE were supported by Swinburne Research, Swinburne University of Technology.

Authors' relationships and activities $\mathrm{GAH}, \mathrm{MCT}$ and $\mathrm{MEC}$ received research support from Boehringer Ingelheim for this project. MCT and MEC have received honoraria for educational meetings and for advisory boards conducted on behalf of pharmaceutical companies involved in treatment with SGLT2 inhibitors including Boehringer Ingelheim, Astra Zeneca, MSD, Lilly and MundiPharma. All other authors declare that there is no conflict of interest with their contribution to this manuscript. Boehringer Ingelheim was involved in study design and approved the decision to submit the report for publication.

Contribution statement All authors contributed to conception and design, acquisition of data, or analysis and interpretation of data; drafting the article or revising it critically for important intellectual content; and final approval of the version to be published. GAH is responsible for the integrity of the work as a whole.

\section{References}

1. Zelniker TA, Wiviott SD, Raz I et al (2019) SGLT2 inhibitors for primary and secondary prevention of cardiovascular and renal outcomes in type 2 diabetes: a systematic review and metaanalysis of cardiovascular outcome trials. Lancet 393(10166):3139. https://doi.org/10.1016/S0140-6736(18)32590-X

2. Fitchett D, Inzucchi SE, Cannon CP et al (2019) Empagliflozin reduced mortality and hospitalization for heart failure across the spectrum of cardiovascular risk in the EMPA-REG OUTCOME trial. Circulation 139(11):1384-1395. https://doi.org/10.1161/ CIRCULATIONAHA.118.037778

3. Huggett RJ, Scott EM, Gilbey SG, Stoker JB, Mackintosh AF, Mary DA (2003) Impact of type 2 diabetes mellitus on sympathetic neural mechanisms in hypertension. Circulation 108(25):30973101. https://doi.org/10.1161/01.CIR.0000103123.66264.FE

4. Rahman A, Fujisawa Y, Nakano D, Hitomi H, Nishiyama A (2017) Effect of a selective SGLT2 inhibitor, luseogliflozin, on circadian rhythm of sympathetic nervous function and locomotor activities in metabolic syndrome rats. Clin Exp Pharmacol Physiol 44(4):522525. https://doi.org/10.1111/1440-1681.12725

5. Burke SL, Head GA (2003) Method for in vivo calibration of renal sympathetic nerve activity in rabbits. J Neurosci Methods 127(1): 63-74. https://doi.org/10.1016/S0165-0270(03)00121-3

6. Zola BE, Miller B, Stiles GL, Rao PS, Sonnenblick EH, Fein FS (1988) Heart rate control in diabetic rabbits: blunted response to isoproterenol. Am J Physiol Endocrinol Metab 255(5 Pt 1):E636E641. https://doi.org/10.1152/ajpendo.1988.255.5.E636

7. Wang J, Wan R, Mo Y, Zhang Q, Sherwood LC, Chien S (2010) Creating a long-term diabetic rabbit model. Exp Diabetes Res 2010: 289614. https://doi.org/10.1155/2010/289614
8. Dorward PK, Riedel W, Burke SL, Gipps J, Korner PI (1985) The renal sympathetic baroreflex in the rabbit. Arterial and cardiac baroreceptor influences, resetting, and effect of anesthesia. Circ Res 57(4):618-633. https://doi.org/10.1161/01.RES.57.4.618

9. Burke SL, Lim K, Moretti JL, Head GA (2016) Comparison of sympathetic nerve activity normalization procedures in conscious rabbits. Am J Physiol Heart Circ Physiol 310(9):H1222-H1232. https://doi.org/10.1152/ajpheart.00866.2015

10. Ricketts JH, Head GA (1999) A five parameter logistic equation for investigating asymmetry of curvature in baroreflex studies. Am J Physiol Regul Integr Comp Physiol 277(2):R441-R454. https://doi. org/10.1152/ajpregu.1999.277.2.R441

11. Head GA, Lukoshkova EV, Burke SL, Malpas SC, Lambert EA, Janssen BJ (2001) Comparing spectral and invasive estimates of baroreflex gain. IEEE Eng Med Biol Mag 20(2):43-52. https:// doi.org/10.1109/51.917723

12. Korner PI, Badoer E, Head GA (1987) Cardiovascular role of the major noradrenergic cell groups in the rabbit: analysis based on 6hydroxydopamine-induced transmitter release. Brain Res 435(1-2): 258-272. https://doi.org/10.1016/0006-8993(87)91609-x

13. Ludbrook J (1994) Repeated measurements and multiple comparisons in cardiovascular research. Cardiovasc Res 28(3):303-311. https://doi.org/10.1093/cvr/28.3.303

14. Nagura S, Sakagami T, Kakiichi A, Yoshimoto M, Miki K (2004) Acute shifts in baroreflex control of renal sympathetic nerve activity induced by REM sleep and grooming in rats. J Physiol 558(Pt 3): 975-983. https://doi.org/10.1113/jphysiol.2004.064527

15. Holwerda SW, Restaino RM, Manrique C, Lastra G, Fisher JP, Fadel PJ (2016) Augmented pressor and sympathetic responses to skeletal muscle metaboreflex activation in type 2 diabetes patients. Am J Physiol Heart Circ Physiol 310(2):H300-H309. https://doi. org/10.1152/ajpheart.00636.2015

16. Straznicky NE, Grima MT, Sari CI et al (2012) Neuroadrenergic dysfunction along the diabetes continuum: a comparative study in obese metabolic syndrome subjects. Diabetes 61(10):2506-2516. https://doi.org/10.2337/db12-0138

17. Ninomiya I, Malpas SC, Matsukawa K, Shindo T, Akiyama T (1993) The amplitude of synchronized cardiac sympathetic nerve activity reflects the number of activated pre- and postganglionic fibers in anesthetized cats. J Auton Nerv Syst 45(2):139-147. https://doi.org/10.1016/0165-1838(93)90125-E

18. Krishnan D, Liu L, Wiebe SA, Casey JR, Cordat E, Alexander RT (2015) Carbonic anhydrase II binds to and increases the activity of the epithelial sodium-proton exchanger, NHE3. Am J Physiol Renal Physiol 309(4):F383-F392. https://doi.org/10.1152/ajprenal. 00464.2014

19. Solomon R, Weinberg MS, Dubey A (1991) The diurnal rhythm of plasma potassium: relationship to diuretic therapy. J Cardiovasc Pharmacol 17(5):854-859. https://doi.org/10.1097/00005344199105000-00023

20. Mazidi M, Rezaie P, Gao HK, Kengne AP (2017) Effect of sodiumglucose cotransport-2 inhibitors on blood pressure in people with type 2 diabetes mellitus: a systematic review and meta-analysis of 43 randomized control trials with 22528 patients. J Am Heart Assoc 6(6):e004007. https://doi.org/10.1161/JAHA.116.004007

21. Zaccardi F, Webb DR, Htike ZZ, Youssef D, Khunti K, Davies MJ (2016) Efficacy and safety of sodium-glucose co-transporter-2 inhibitors in type 2 diabetes mellitus: systematic review and network meta-analysis. Diabetes Obes Metab 18(8):783-794. https://doi.org/10.1111/dom.12670

22. Kusaka H, Koibuchi N, Hasegawa Y, Ogawa H, Kim-Mitsuyama S (2016) Empagliflozin lessened cardiac injury and reduced visceral adipocyte hypertrophy in prediabetic rats with metabolic syndrome. Cardiovasc Diabetol 15(1):157. https://doi.org/10.1186/s12933016-0473-7 
23. Thomas MC, Cherney DZI (2018) The actions of SGLT2 inhibitors on metabolism, renal function and blood pressure. Diabetologia 61(10):2098-2107. https://doi.org/10.1007/s00125-018-4669-0

24. Wanner C, Inzucchi SE, Lachin JM et al (2016) Empagliflozin and progression of kidney disease in type 2 diabetes. N Engl J Med 375(4):323-334. https://doi.org/10.1056/NEJMoa1515920

25. Zinman B, Wanner C, Lachin JM et al (2015) Empagliflozin, cardiovascular outcomes, and mortality in type 2 diabetes. N Engl J Med 373(22):2117-2128. https://doi.org/10.1056/ NEJMoa1504720

26. Seravalle G, Mancia G, Grassi G (2014) Role of the sympathetic nervous system in hypertension and hypertension-related cardiovascular disease. High Blood Press Cardiovasc Prev 21(2):89105. https://doi.org/10.1007/s40292-014-0056-1
27. Masuo K, Lambert GW, Esler MD, Rakugi H, Ogihara T, Schlaich MP (2010) The role of sympathetic nervous activity in renal injury and end-stage renal disease. Hypertens Res 33(6):521-528. https:// doi.org/10.1038/hr.2010.35

28. Perin PC, Maule S, Quadri R (2001) Sympathetic nervous system, diabetes, and hypertension. Clin Exp Hypertens 23(1-2):45-55. https://doi.org/10.1081/CEH-100001196

29. Jordan J, Tank J, Heusser K et al (2017) The effect of empagliflozin on muscle sympathetic nerve activity in patients with type II diabetes mellitus. J Am Soc Hypertens 11(9):604-612. https://doi.org/10. 1016/j.jash.2017.07.005

Publisher's note Springer Nature remains neutral with regard to jurisdictional claims in published maps and institutional affiliations. 